\title{
Gut check: dead cell samples leads to tolerant examples
}

\author{
Thomas H Oguin III ${ }^{1}$ and Jennifer Martinez ${ }^{\star, 1}$
}

Cell Death and Differentiation (2017) 24, 1471-1472; doi:10.1038/cdd.2017.45; published online 23 June 2017

Recent studies have garnered attention and appreciation for the role that phagocytes of the innate immune system play in the clearance of dying cells, a process termed efferocytosis. The sensing, recognition, and removal of cellular corpses is a reoccurring event throughout the lifetime of organisms, and considering the billions of cells that undergo apoptosis daily and the rarity of observing an uncleared apoptotic cell under normal physiological conditions, one must truly admire the efficiency with which phagocytes perform their duties. ${ }^{1}$ The clearance of dead cells is designed to be a tolerated mechanism, as apoptosis is a common, genetically programmed process designed to sculpt, control and aid the body in its development and survival. ${ }^{2}$ Thus, efferocytosis must occur in an 'immunologically silent' manner, so as to not inappropriately alert the immune system during times of development, cellular homeostasis, and stress. ${ }^{3}$

However, not all tissue-resident phagocytes are created equally when it comes to efferocytosis. Words such as 'phagocytes' and 'tissue' are broad terms that minimize the uniqueness and specificity that underlies each cellular subset within individual tissue environments. Phagocytes within the skin do not encounter the same challenges as phagocytes in the gut, and different types of phagocytes within the same tissue are evolutionarily poised to carry out certain distinct functions. ${ }^{4}$ Cummings et al. develop an exciting mouse model capable of inducing tissue-specific apoptosis and to track the fate of the phagocytes that efferocytose these corpses. This novel mouse model, termed the VDTR mouse, expresses a transgenic diphtheria toxin receptor fused to eGFP under the control of the epithelial cell specific villin promoter. After administration of a non-inflammatory dose of diphtheria toxin, the intestinal epithelial cells (IECs) in the small intestine of the VDTR mouse undergo apoptosis, as determined by caspase3 cleavage. As the apoptotic cells express eGFP, their fate and influence can be followed with the phagocytic subsets that engulf and process them. ${ }^{5}$

The authors use this innovative model to ask the long unanswered question of what subset(s) of phagocytes sample dying cells and how do these subsets within a tissue differ in terms of their response (Figure 1). First, the authors demonstrate that the tissue-resident phagocytes, not recruited phagocytes, were responsible for apoptotic cell eating in the small intestine. These data lends credence to our new understanding that 'find-me' signals, such as S1P, released by dying cells function less as chemoattractants, and more as factors that prime phagocytes for efferocytosis locally. ${ }^{6}$

Which resident phagocytes heed the call when apoptosis occurs in the small intestine? Using flow cytometry and ImageStream technology, the authors find that out of the five phenotypically distinct phagocytes in small intestine lamina propria (SILP), only one dendritic cell subset (CD103 ${ }^{+} \mathrm{CD} 24^{+}$ $\mathrm{CD}^{-} 4^{-}$) and two macrophage subsets (CD24 $4^{-} \mathrm{CD} 64^{+} \mathrm{CD} 103^{+}$ $\mathrm{CD} 11 \mathrm{~b}^{+}$and $\mathrm{CD} 24^{-} \mathrm{CD}^{+} 4^{+} \mathrm{CD} 103^{-} \mathrm{CD} 11 \mathrm{~b}^{+}$) were responsible for the vast majority of efferocytosis, and this sampling occurred independently of the lumen, as is seen during soluble antigen removal. Interestingly, these phagocytes were also noted to carry fluorescent apoptotic cargo in untreated VDTR mice, demonstrating the clearance of dead cells in steady state conditions, as well as in experimental conditions.

Despite similar capacity to recognize and engulf apoptotic IECs, these phagocytic subsets were transcriptionally distinct upon dead cell ingestion, suggesting that a phagocyte's response during efferocytosis is tailored to perform its unique function. Overall, all three phagocytic subsets generally demonstrated a transcriptional profile consistent with the anti-inflammatory state, but the differential regulation of specific genes within each cell type highlighted the important role that each plays in achieving this tolerant goal. ${ }^{7,8}$ Macrophages have historically been considered the predominant cell type for physical clearance of dying cells, and both subsets of macrophages upregulated genes critical for apoptotic cargo internalization (such as Gas6, Mertk, Axl, Stab1, Bai1, and Tim4d) and degradation (such as Pikfyve). Metabolic mechanisms by which a phagocyte handles these ingested corpses is an area of growing interest. CD103 ${ }^{+}$ $\mathrm{CD}_{11} \mathrm{~b}^{+}$macrophages upregulated genes involved in lipid metabolism and cholesterol efflux, whereas CD103- CD11 ${ }^{+}$ macrophages upregulated those involved in branched-chain amino acid catabolism. ${ }^{9}$ Concurrent with these upregulations were the downregulation of pro-inflammatory mediators, such as TIr2, Clec4a, and Cd209a.

The $\mathrm{CD} 103^{+}$dendritic cell subset responded with a transcriptional profile, unique from that of the macrophage subsets. Uptake of apoptotic IECs also triggered an

\footnotetext{
${ }^{1}$ Immunity, Inflammation, and Disease Laboratory, National Institute of Environmental Health Sciences, National Institutes of Health, Research Triangle Park, NC 27709, USA

*Corresponding author: J Martinez, Immunity, Inflammation, and Disease Laboratory, National Institute of Environmental Health Sciences, National Institutes of Health, 111 T.W. Alexander Drive, Research Triangle Park, NC 27709, USA. Tel: +1 919541 4420; E-mail: jennifer.martinez3@nih.gov
} 


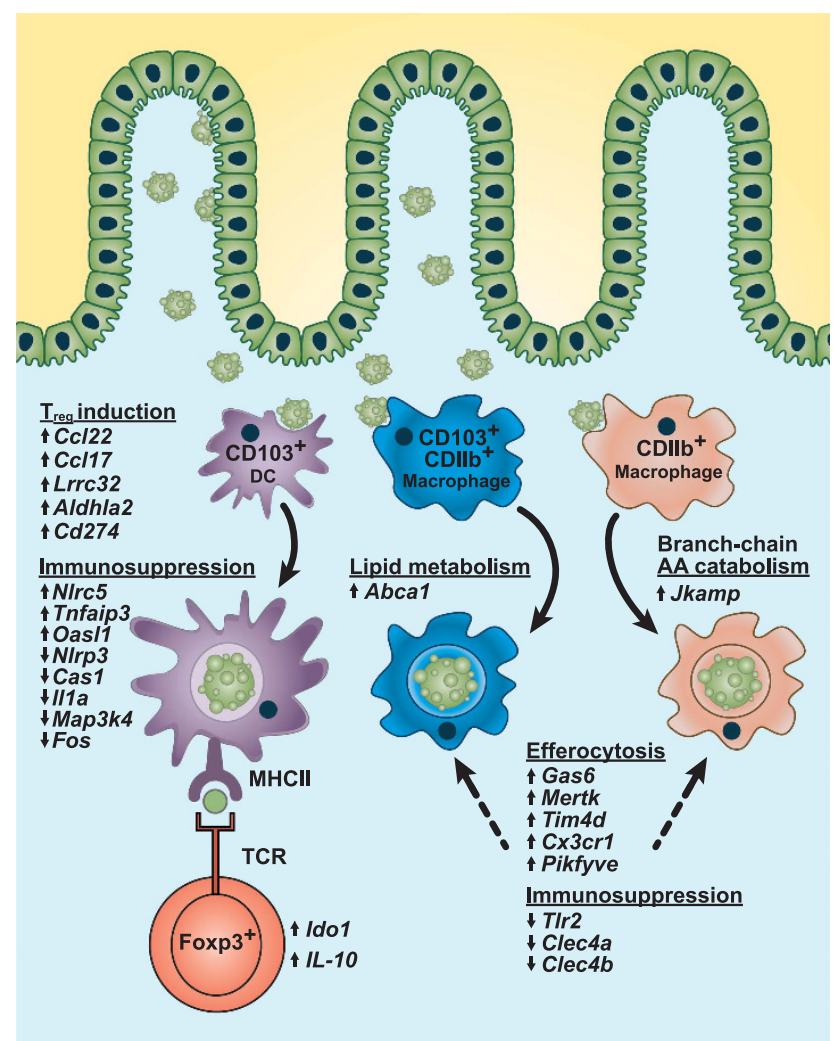

Figure 1 Efferocytosis induces cell type-specific programming. In Cummings et al., the authors use the novel VDTR mouse to demonstrate that uptake of apoptotic IECs triggers a differential transcriptional response in a cell specific manner. Two macrophage subsets (CD103 ${ }^{+}$and $\mathrm{CD}_{103^{+}} \mathrm{CD} 11 \mathrm{~b}_{+}$macrophages) phagocytose IECs and upregulate transcriptional programs associated with dead cell clearance, immunosuppression, lipid metabolism, branch-chain amino acid (AA) catabolism. $\mathrm{CD}_{103^{+}}$dendritic cells (DC), on the other hand, promote immunosuppression and upregulate molecules critical for the recruitment, differentiation, and activity of regulatory $T$ cells $\left(T_{\text {reg }}\right)$

immunosuppressive program in $\mathrm{CD}_{103^{+}}$dendritic cells, although the repertoire of genes affected were distinct from those affected in macrophages. The dendritic cell subset downregulated components of the inflammasome pathway (NIrp3, Casp1, and II1a) and MAPK/ERK signaling (Lrrk2, Map3k4 and Fos), while simultaneously upregulating negative regulators of inflammation (Cfh, Tnfaip3, Oas/1 and Mtor). One of the most well-defined roles for dendritic cells is the presentation of antigen to $\mathrm{T}$ cells to mediate their activation. Consistent with an overall anti-inflammatory profile, CD103 ${ }^{+}$ dendritic cells were enriched for changes that promoted $\mathrm{T}_{\text {reg }}$ recruitment (Cc122 and $C c / 17)$, differentiation (Aldh1a2), and activity (Lrrc32 and Cd274). ${ }^{10}$ These CD103 ${ }^{+}$dendritic cells were also readily observed in the mesenteric lymph node (MLN), where they had a similar, anti-inflammatory transcriptional profile. Transcripts such as $1 / 10$, Ido, and Itgb8 were additionally increased in MLN $\mathrm{CD}_{103^{+}}$dendritic cells compared to SILP counterparts. This collective increase in $T_{\text {reg }}$ differentiation capacity resulted in an increase in $T_{\text {reg }}$ induction, both in vivo and ex vivo.

Inflammatory bowel disease (IBD) is a persistent inflammatory disease of the digestive tract that affects over 1 million people in the United States and over 2.5 million people in
Europe. ${ }^{11}$ To date, 163 susceptibility loci have been identified via GWAS studies on IBD, and the authors identified 41 overlapping genes that were modulated in response to apoptotic IECs. However, the so-called sterile inflammation created by apoptosis does not fully recapitulate the chronic inflammation associated with IBD. Treatment of VDTR mice with DSS resulted in downregulation of many of the $T_{\text {reg }}$ activating genes, such as Aldh1a2, Ccl22, Cd274, Ido1, and I/10, suggesting that an inflammatory environment during dead cell clearance can have dramatic effects on the function of efferocytotic phagocytes.

Investigating the molecular mechanisms of efferocytosis that mediate immunosuppression from the digestive tract could lead to discoveries that further the understanding of the etiology and treatment of different inflammatory conditions. Taken together, the VDTR mouse model allows for fine dissection of the dynamics of dead cell clearance in the mouse gut in both homeostatic or pathologic settings, and can serve as a template for exploring the role and function of different phagocytes in different tissue environments and during homeostatic or inflammatory diseases. IBD itself has undergone a remarkable globalization, and the crosstalk between genetics, the gut microbiome, and environment are currently an area of fast paced exploration. We now appreciate the distinct microbiotic niche that exists within different tissues, such as the lung, replete with its own specialized flavors of phagocytes. ${ }^{12}$ This novel mouse model has opened the door on determining how these subsets sample their environment, and how that sampling leads to immune modulation. An area of growing interest is the molecular program that dictates how a phagocyte handles and processes an ingested apoptotic cell, wherein each specific subset is predisposed to differential response.

Finally, at the center of this paradigm is the cellular corpse. Like phagocytes, cellular corpses are not created equally, and most modes of cell death do not promote a tolerant environment. The role that programmed necrosis or necroptosis plays in infectious immunity and cancer is an evolving field, where the field is tasked with determining how we can harness this inflammatory, yet malleable form of cell death in a manner that lends itself to therapeutic intervention. ${ }^{13}$ In such an inflammatory environment, it is unknown if the transcriptional gap that separates cellular subsets will narrow, in an effort to combine forces for the greater good of immunosuppression.

\section{Conflict of Interest}

The authors declare no conflict of interest.

1. Arandjelovic S et al. Nat Immunol 2015; 16: 907-917.

2. Green DR. Immunity 2011; 35: 441-444.

3. Nagata S. Ann N Y Acad Sci 2010; 1209: 10-16.

4. Cummings RJ et al. Nature 2016; 539: 565-569.

5. Poon IK et al. Nat Rev Immunol 2014; 14: 166-180.

6. Luo B et al. Immunity 2016; 44: 287-302.

7. Liu K et al. J Exp Med 2002; 196: 1091-1097.

8. Zizzo G et al. J Immunol 2012; 189: 3508-3520.

9. Martinez J et al. Cell Metab 2013; 17: 895-900.

10. Vignali DA et al. Nat Rev Immunol 2008; 8: 523-532.

11. Cho JH. Nat Rev Immunol 2008; 8: 458-466.

12. Blaser MJ. J Clin Invest 2014; 124: 4162-4165.

13. Weinlich R et al. Nat Rev Mol Cell Biol 2017; 18: 127-136. 